\title{
Implemention of a Laboratory Information System in Zimbabwe
}

\author{
Rita Sembajwe ${ }^{1}$, Tendai Shamu ${ }^{\star 2}$, Fortunate Machingura ${ }^{1}$ and Henry Chidawanyika ${ }^{1}$
}

${ }^{1}$ RTI International, Decatur, GA, USA; ${ }^{2}$ Zimbabwe Ministry of Health and Child Care, Harare, Zimbabwe

\section{Objective}

Understand the challenges that exist in the Zimbabwe health systems, that could be addressed through the integration of a Laboratory Information Management System (LIMS).

Understand key aspects for consideration when selecting and adapting a LIMS in a resource limited setting.

Showcase improvements in laboratory information management processes following adoption of a LIMS.

\section{Introduction}

Zimbabwe's National Health Laboratory Services faces multiple challenges related to inadequate financial support and skilled human resources, insufficient infrastructure, and inefficient tracking of clinical samples collected by health facilities. The slow turnaround time and poor management of the sample testing process, as well as delivery of results remain critical challenges. Compounding these problems further is a manual system for tracking large volumes of samples. This laborious and time-consuming process is inefficient for management of high amounts of incoming medical samples, frequently resulting in incomplete and inaccurate data. Additionally, health facilities are unable to monitor clinical samples and results in transit, leading to misplaced samples and missing results. Furthermore, although the laboratory service runs on a tiered network system - with lower level laboratories referring surveillance samples to higher level laboratories, processing of samples is not fulfilled promptly. The solutions to these challenges are divergent - sometimes even pointing in different directions. To this end, the Zimbabwe Ministry of Health and Child Care (MoHCC) has identified and integrated a LIMS to improve tracking of samples from the time of collection through results delivery.

\section{Methods}

Our methods included an environmental needs assessment, user requirement analysis, followed by a LIMS customization and integration. The overarching aim has been to integrate the electronic open source BIKA LIMS into Zimbabwe's national health information systems (HIS), to improve laboratory information management. The user requirements gathering exercise, included focus group discussion meetings with potential LIMS users, and direct observations, to guide the establishment of LIMS specifications. The needs assessment focused on the system functionality. Specifically, it investigated those aspects that would improve the ability: to track clinical samples such as creating and activating an 'alerting' capability when results are not reported within the set turnaround time; for users to see lists and counts of clinical samples at various testing levels; to uniquely identify samples received in the laboratories. Guided by these requirements, an environmental scan of off-the-shelf and open source LIMS platforms was conducted to identify a few options for the Zimbabwe context. Primary factors for shortlisting included: an existing community of practice for support; interoperability; customizability and configurability; and local awareness of the platform. In a LIMS national user's meeting, involving relevant levels of the health system (Laboratories, Central, Provincial and District hospitals), a review of LIMS platform options was performed to narrow down selections. It evaluated the extent to which the user requirements (Workflow, equipment interface, result management, inter-operable, quality control, and stock management) were being met. Based on the evaluation, a single system (LIMS) was selected, adopted and adapted for use at six representative laboratories, including Zimbabwe's National Microbiology Reference Laboratory.

On-Site classroom and desk-side training, for knowledge transfer to local LIMS users, characterised the implementation phase. Local champions were identified from laboratory technicians and equipped to offer first line support. Both on-site and remote support was provided to LIMS users. The monitoring phase is ongoing, using interview guides and LIMS user meetings to understand challenges and ways to improve the system.

\section{Results}

A LIMS was successfully customized and integrated into Zimbabwe's national health information system infrastracture in six regional laboratories, to improve overall laboratory information management, timeliness of reporting and quality control. Since its full implementation between 2013 and 2017, average turnaround time for results improved significantly from 10 to 21 days in 2013 to only 3 days in 2017. Data quality improved; the number of untested clinical samples reduced from an average of 6 in 100 in 2013, to average of less or equal to 1 in 100, in 2017. Also, there have been observed improvements in Zimbabwe's laboratory information management workflow and results reporting. High user satisfaction and increased LIMS use have led to the demand for LIMS expansion to additional laboratories. The LIMS has also managed to reduce the time required to produce disease notification reports.

\section{Conclusions}

LIMS are proving to be an effective method for tracking samples and laboratory results in low resource settings like Zimbabwe. LIMS has provided an efficient way for record, store, and track timely reporting of laboratory data, allowing for improved quality of data. Overall, LIMS has increased efficiency in laboratory workflow and introduced the ability to adequately track samples from time of collection.

\section{Keywords}

laboratory information systems; health information systems; health informatics; Laboratory Information Management Systems

\section{Acknowledgments}

This work falls under a project that has been supported by the U.S President's Emergency Plan for AIDS Relief, through the U.S. Centers for Disease Control and Prevention.

\section{References}

Southern Africa Development Community (SADC). 2009. Assessment Report on Reference Laboratories in the SADC Region. Gaborone: Directorate of Social and Human Development And Special Programs, SADC Secretariat

\section{*Tendai Shamu}

E-mail: shamut@nmrl.org.zw 